\title{
Cone pigments in a North American marsupial, the opossum (Didelphis virginiana)
}

\author{
Gerald H. Jacobs • Gary A. Williams
}

Received: 28 January 2010/Revised: 26 February 2010/Accepted: 2 March 2010/Published online: 12 March 2010

(C) The Author(s) 2010. This article is published with open access at Springerlink.com

\begin{abstract}
Only two of the four cone opsin gene families found in vertebrates are represented in contemporary eutherian and marsupial species. Recent genetic studies of two species of South American marsupial detected the presence of representatives from two of the classes of cone opsin genes and the structures of these genes predicted cone pigments with respective peaks in the ultraviolet and long-wavelength portions of the spectrum. The Virginia opossum (Didelphis virginiana), a profoundly nocturnal animal, is the only marsupial species found in North America. The prospects for cone-based vision in this species were examined through recordings of the electroretinogram (ERG), a commonly examined retinal response to photic stimulation. Recorded under flickering-light conditions that elicit signals from cone photoreceptors, the spectral sensitivity of the opossum eye is well accounted for by contributions from the presence of a single cone pigment having peak absorption at 561-562 nm. A series of additional experiments that employed various chromatic adaptation paradigms were conducted in a search for possible contributions from a second (short-wavelength sensitive) cone pigment. We found no evidence that such a mechanism contributes to the ERG in this marsupial.
\end{abstract}

Keywords Cone pigments - Marsupials ·

Didelphis virginiana $\cdot$ Electroretinogram

G. H. Jacobs $(\bowtie)$

Department of Psychology, University of California,

Santa Barbara, CA 93106, USA

e-mail: jacobs@psych.ucsb.edu

G. A. Williams

Department of Psychology and Child Development,

California State University Stanislaus, Turlock, CA 95382, USA

\section{Introduction}

In recent years direct measurements of cone pigments combined with inferences drawn from examinations of the genes coding for photopigment opsins have provided a greatly enhanced understanding of the distribution and evolution of cone photopigments. The consensus is that four families of genes specify the opsin proteins in all vertebrate cone pigments (Yokoyama 2000, 2008; Hisatomi and Tokunaga 2002). Eutherian mammals have retained representatives of only two of these-SWS1 genes linked to pigments with peak absorbance $\left(\lambda_{\max }\right)$ in the ultraviolet (UV) and short-wavelength (S) portions of the visible spectrum (360-445 nm) and LWS genes that yield pigments with $\lambda_{\max }$ in the middle to long wavelengths (M/L $500-565 \mathrm{~nm}$ ). Most species of this group derive one type of cone pigment from each of these two classes providing the potential for a dichromatic color vision capacity (Jacobs 2009). There are notable exceptions to this arrangement among the primates, a group in which LWS opsin gene duplications and polymorphisms have allowed many lineages to gain a total of three cone photopigments and from these derive trichromacy (Jacobs 2008).

There has also been progress toward understanding the cone pigments of protherian and metatherian mammals. Among the handful of contemporary monotremes, two cone opsin gene families have been detected in both platypus (Ornithorhynchus) and echidna (Tachyglossus) (Davies et al. 2007; Wakefield et al. 2008) The opsin genes in these animals are drawn from the SWS2 and LWS groups and yield cone pigments with respective $\lambda_{\max }$ values of 450 and $550 \mathrm{~nm}$. The cone pigment arrangements of marsupials are more varied. Some species of Australian marsupials have two classes of cone pigments, while others have three (Hemmi 1999; Hemmi et al. 2000; Arrese et al. 
2002, 2005). Among the examples of latter is the fat-tailed dunnart (Sminthopsis carssicaudatus). Direct pigment measurements in this animal revealed three types of cone pigment and subsequent behavioral measurements have verified the presence of a trichromatic color vision capacity (Arrese et al. 2006). However, whereas two of these cone pigment types are representatives of the SWS1 and LWS gene families, there is no direct evidence that the third cone pigment $\left(\lambda_{\max }\right.$ of $\left.\sim 500 \mathrm{~nm}\right)$ in fact derives from a cone opsin gene (Strachan et al. 2004; Cowing et al. 2008). To this point the gene/cone pigment linkage in this marsupial therefore remains somewhat puzzling, but one possibility is that the third type of cone in this animal contains a rod photopigment.

Roughly one-third of all marsupial species are found in the Americas. A vast majority of these are native to South America and recent examination of the opsin genes in two species from this group (Didelphis auria, the bigeared opossum, and Monodelphis domestica, the gray short-tailed opossum) detected opsin genes for two types of cone photopigment drawn, respectively, from SWS1 and LWS gene families (Hunt et al. 2009). Opossums are believed to have migrated northward from South America across the Isthmus of Panama perhaps about 3 million years ago (Macdonald 2001). Only a single species, the Virginia opossum (D. virginiana), is found in North America where it currently occupies a range that runs northward from Mexico to the Canadian border encompassing much of the eastern two-thirds of the United States as well as the western states bordering the Pacific ocean. This species is stringently nocturnal and in accord with that behavior the retina contains only a sparse population of retinal cones (Walls 1939; Wikler and Rakic 1990; Kolb and Wang 1985). Over the years, we have employed a gross potential electrophysiological technique to attempt to access cone signals in the retina of this species (some early efforts were noted in Jacobs 1993). Because of the current interest in understanding the nature of marsupial cone pigments, a brief summary of the more recent results from these efforts is provided here.

\section{Materials and methods}

Adult Virginia opossums (D. virginiana) of both sexes were trapped in Santa Barbara County, California under the auspices of a scientific collecting permit issued by the State of California, Department of Fish and Game. All animal care and experimental procedures were in accordance with institutional animal care and use guidelines and with the Guide for the Care and Use of Laboratory Animals (National Academy Press, 1996).
In preparation for recording the opossums were anesthetized with a mixture of xylazine hydrochloride $\left(8 \mathrm{mg} \mathrm{kg}^{-1}\right)$ and ketamine hydrochloride $\left(42 \mathrm{mg} \mathrm{kg}^{-1}\right)$ injected intramuscularly followed by intraperitoneal administration of pentobarbital sodium $\left(50 \mathrm{mg} \mathrm{kg}^{-1}\right)$. The pupil of one eye was dilated by topical application of atropine sulfate $(0.04 \%)$. The animal was positioned in a head holder and aligned with a three-beam Maxwellian view optical system that images a $59^{\circ}$ circular field on the retina. Hypothermia has been reported to be a major side effect of anesthesia in opossums (Keller et al. 1988) so throughout the recording session body temperature was supported with the use of a circulating hot water heater. The electroretinogram (ERG) was differentially recorded using a contact lens electrode. To enhance the likelihood of eliciting contributions from cones the recording sessions were conducted in a room that was ambiently illuminated to yield $\sim 150$ lux at the cornea of the eye being tested.

For recording we employed ERG flicker photometry, a technique frequently used to access cone signals (Jacobs et al. 1996). With this technique, a flickering monochromatic test light (derived from a $15-\mathrm{nm}$ bandpass monochromator supplied with either a $100 \mathrm{~W}$ tungsten-halide lamp or a $150 \mathrm{~W}$ xenon arc lamp) is continuously adjusted in intensity until the fundamental component of the ERG, it elicits is identical to that obtained in response to an interleaved, similarly flickering, reference light. The comparison of the responses to the test and reference lights is based on averages of the last 50 of a total of 70 responses elicited by each light. The reference light is derived from a $100 \mathrm{~W}$ tungsten-halide lamp with test and reference lights aligned so that they optically superimpose. A third stimulus beam, used to provide accessory adaptation, also originates from a $100 \mathrm{~W}$ tungsten-halide lamp and is optically imaged coincident with the other two beams. Further details of the stimuli used in these experiments are noted below as they become appropriate.

\section{Results}

Although ERGs could be readily recorded from the eye of the opossum, cone-based signals were quite small relative to those we have recorded from the eyes of a number of other strongly nocturnal mammals using similar techniques, e. g., two species of procyonids (Jacobs and Deegan 1992). Using a tungsten-halide lamp as the monochromator source, it proved possible to measure spectral sensitivity functions in $10 \mathrm{~nm}$ steps across the spectrum from 460 to $650 \mathrm{~nm}$. For these measurements, the flicker rate was $20 \mathrm{~Hz}$ and the reference light was achromatic $(2,850 \mathrm{~K})$ yielding a retinal illuminance of $2.7 \mathrm{log}$ td. All wavelengths were tested twice and the resulting photometric 
equation values were subsequently averaged. The averaged spectral sensitivity values $( \pm 1 \mathrm{SD})$ so obtained for seven opossums ( 5 males, 2 females) are shown in Fig. 1. It is apparent that despite the small amplitudes of the cone signals the variability in spectral sensitivity across the sample is quite small. The full array of sensitivity values was best fit using a standard visual pigment template (Govardovskii et al. 2000) that was shifted along the wavelength axis in steps of $0.1 \mathrm{~nm}$ to find the spectral location that provided the best least squares fit to the full data array. A template (shown as the continuous line in Fig. 1) having $\lambda_{\max }=562.4 \mathrm{~nm}$ provides a good account of the averaged data. For similar fits made to the data arrays obtained from the seven animals individually, the mean $\lambda_{\text {max }}$ was $561.4 \mathrm{~nm}(\mathrm{SD}=1.78)$.

In an attempt to extend the spectral sensitivity measurements to encompass a greater spectral span a xenon lamp was substituted as the light source for the monochromator. All other details were as described above. The xenon light source allowed measurement of spectral sensitivity to be made in $10 \mathrm{~nm}$ steps from 370 to $670 \mathrm{~nm}$. The results obtained from a single animal are shown in Fig. 2. As before, the resulting sensitivity measurements were best fit using a visual pigment template. The estimated peak is close to that for the more spectrally truncated function of Fig. 1 -in this case having a $\lambda_{\max }$ of $561.6 \mathrm{~nm}$. Note that the template provides a reasonable account of the entire span of spectral sensitivity and that, in particular, there is no obvious elevation of sensitivity in the short wavelengths beyond that predicted by contribution from a single type of photopigment.

ERG spectral sensitivity functions recorded from opossums suggest the retina of this marsupial contains a cone

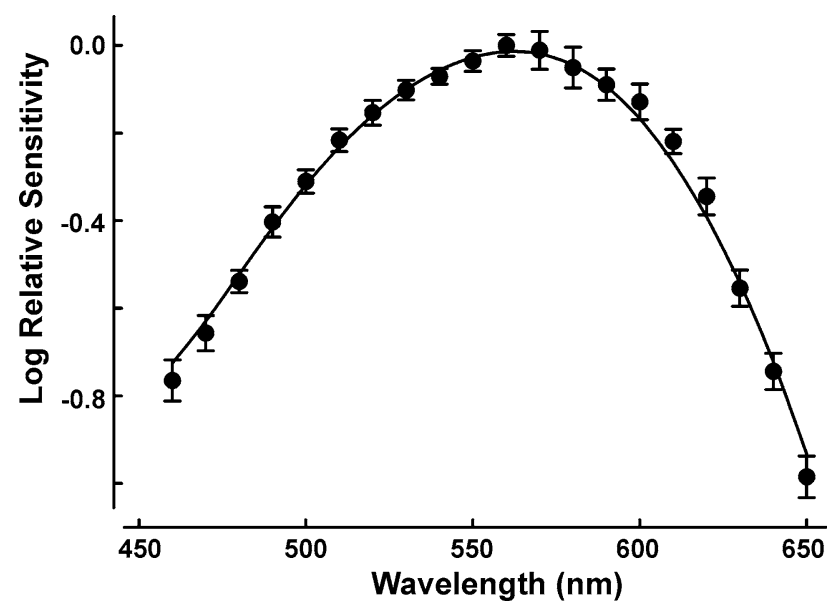

Fig. 1 Spectral sensitivity of the Virginia opossum ERG measured for test conditions designed to capture cone signals. The solid circles are mean values for seven animals $( \pm 1 \mathrm{SD})$. These values were best fit to a visual pigment absorption function having a peak value of $562.4 \mathrm{~nm}$ (continuous line). Details of the test conditions and fitting procedure are given in the text

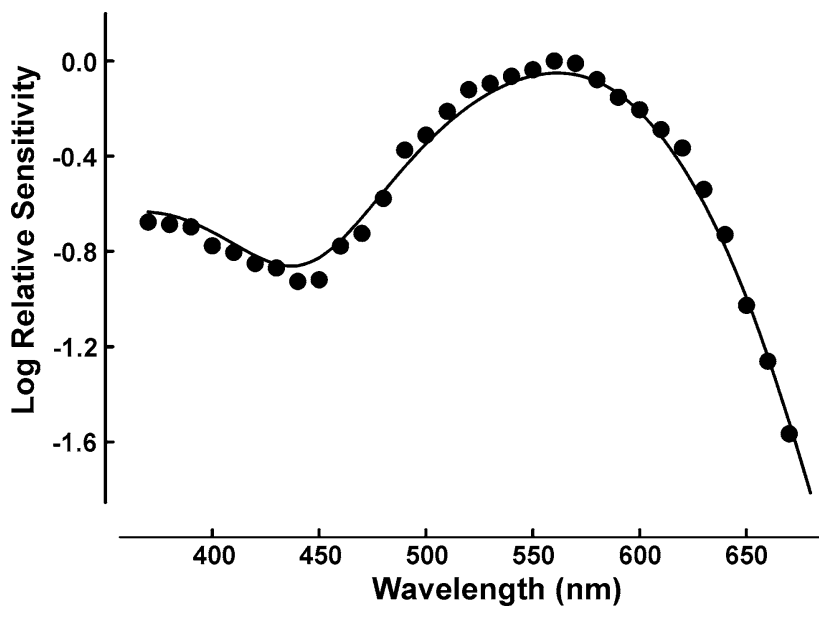

Fig. 2 Extended cone spectral sensitivity of the ERG of the Virginia opossum. The solid circles are values obtained from a single animal. These have been best fit to a visual pigment absorption function having a peak value of $561.6 \mathrm{~nm}$ (continuous line). Other details are specified in the text

pigment with a peak value of $\sim 561-562 \mathrm{~nm}$, but they give no obvious indication for the presence of any additional types of cone pigment. It is a standard assumption that if only a single pigment contributes to the spectral sensitivity then that spectral sensitivity curve should behave univariantly in the presence of chromatic adaptation (Rushton 1972). We conducted numerous experiments in an attempt to evaluate this possibility. In each of these cases, we measured some aspect of spectral sensitivity while the retina was concurrently chromatically adapted.

No indications of a failure of univariance were detected. Three separate examples that support this conclusion are shown in Fig. 3. In one case, a complete spectral sensitivity function (from 390 to $670 \mathrm{~nm}$ ) was recorded using ERG flicker photometry under the conditions specified above. The function was then re-measured in the identical fashion except in the second instance the eye was concomitantly exposed to a long-wavelength light (produced by placing a long-pass filter having 50\% transmission at $570 \mathrm{~nm}$ into the adaptation beam that produces a light appearing bright orange to a human). The effectiveness of this manipulation was established by determining the intensity of a $610 \mathrm{~nm}$ test light required to produce an ERG having a mean amplitude of $1.5 \mu \mathrm{V}$, first under neutral adaptation and then again in the presence of the orange adaptation light. So measured, this adaptation light produced a $0.9 \log$ unit decrease in sensitivity. Figure $3 \mathrm{a}$ plots the wavelength-by-wavelength differences in sensitivity measured under the two test conditions as established by ERG flicker photometry. The variations in sensitivity are quite small $(0.15 \log$ units or less at all of the test wavelengths) and these variations show no systematic pattern across the spectrum, counter to what would be expected if more than one pigment contributed to the recorded ERG. 

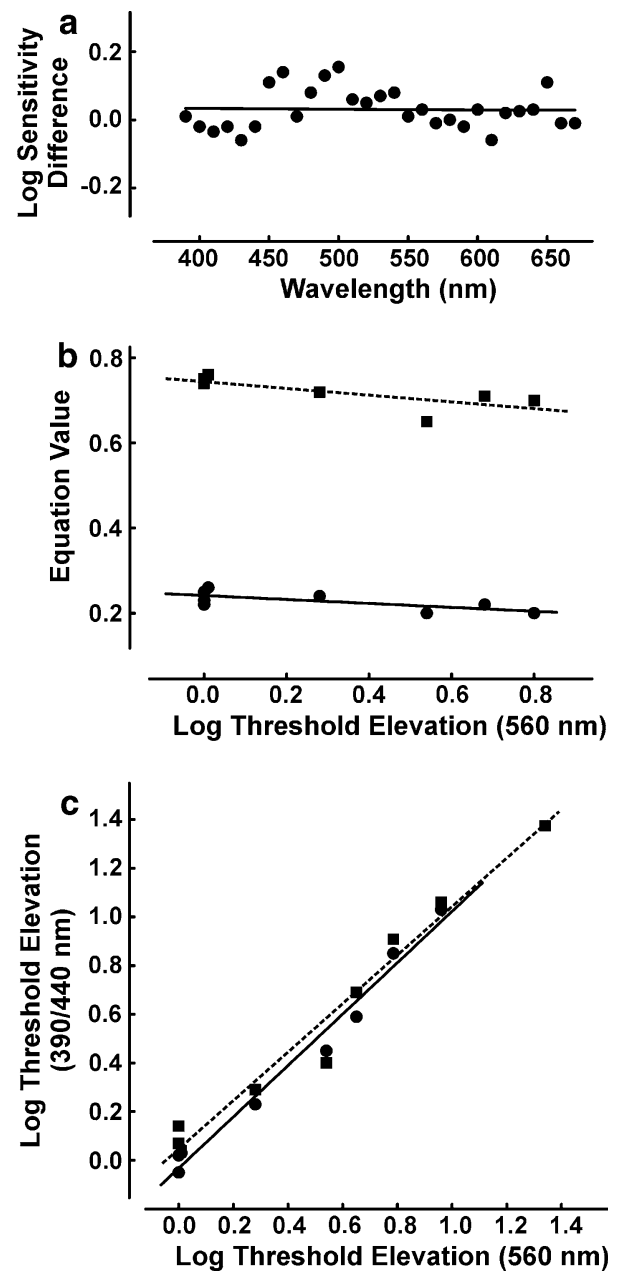

Fig. 3 Results from three separate tests to assess whether the opossum cone ERG behaves univariantly in the face of concurrent chromatic adaptation. a Wavelength-by-wavelength differences (in $\log$ units) in spectral sensitivity obtained from measurements made in the neutrally adapted eye and then repeated as the eye was concurrently adapted to a bright $570 \mathrm{~nm}$ light. The best-fit regression (continuous line) indicates that there is no systematic change in spectral sensitivity between the two test conditions $\left(r^{2}=0.0007\right.$, $P>0.05)$. b ERG flicker photometric equations obtained between a $560 \mathrm{~nm}$ reference light and two test lights (squares $440 \mathrm{~nm}$, circles $390 \mathrm{~nm}$ ). The equations were measured first in the absence of chromatic adaptation and then under increasing levels of chromatic adaptation $(560 \mathrm{~nm})$. The results are plotted as a function of the threshold elevation produced for the reference light and they show no systematic change with chromatic adaptation $\left(440 \mathrm{~nm}, r^{2}=0.37\right.$, $\left.P=0.31 ; 390 \mathrm{~nm}, r^{2}=0.54, P=0.38\right)$. c Threshold elevations obtained for three test lights $(560,440,390 \mathrm{~nm})$ induced by increasing levels of concurrent, long-wavelength adaptation. The results for the latter two (squares and circles, respectively) are plotted as a function of the threshold elevation for the $560 \mathrm{~nm}$ light. The bestfit regressions have respective slopes of $0.98(440 \mathrm{~nm})$ and 1.05 $(390 \mathrm{~nm})$ with $r^{2}$ values for both $=0.98$. Neither of the two are significantly different from a theoretical prediction of a slope of 1.0: $390 \mathrm{~nm} t(6) 7.365, P=0.489 ; 440 \mathrm{~nm}, t(7)=0.115, P=0.911$. Additional details for all three experiments are given in the text
A second type of experiment yielded a very similar outcome. In this case, ERG flicker photometric equations were made between a $560 \mathrm{~nm}$ reference light and two different test lights (440 and $390 \mathrm{~nm}$ ). These test wavelengths were chosen as being near optimal for detecting the presence of contributions from cones containing either UV or S pigment. Equations were made first in the absence of any adaptation light and then repeatedly in the presence of an orange adaptation light (derived as above). The effectiveness of the adaptation was established by determining at each adaptation light level the intensity of a $560 \mathrm{~nm}$ light required to yield a signal having criterion amplitude of $1 \mu \mathrm{V}$. Figure $3 \mathrm{~b}$ shows the result for one animal, but others behaved similarly. Across a range of adaptation light intensity up to a level sufficient to raise the threshold of the reference light by $\sim 0.8 \log$ units there is simply no change in the equation values, i.e., the relative sensitivity at the three wavelengths is unaffected by chromatic adaptation. In a third attempt, thresholds were measured for three test wavelengths $(390,440$, and $560 \mathrm{~nm})$ in the presence of progressively increasing levels of long-wavelength adaptation (produced as described above). The thresholds were defined using criterion amplitudes of $0.8 \mu \mathrm{V}$ for the averaged response to $20 \mathrm{~Hz}$ flicker. The results are shown in Fig. 3c, where threshold elevations measured for the two shorter test lights ( 390 and $440 \mathrm{~nm}$ ) are plotted against the corresponding threshold elevation recorded for the $560 \mathrm{~nm}$ test. Thresholds for the former two do not differ significantly from those recorded to the latter. In sum, in all three of these experiments, we found no changes in spectral sensitivity as would be predicted by a failure of univariance and thus no evidence that more than a single cone type contributes to the photopic ERG in this marsupial.

\section{Discussion}

The retina of the Viriginia opossum has long been known to have an abundant population of rods containing a typical rhodopsin $\left(\lambda_{\max }=\sim 493 \mathrm{~nm}\right)$ (Crescitelli 1958), but there are apparently no previous measurements of cone pigments in this species. As noted, Hunt et al. have recently examined the opsin genes of two species of South American opossums, including one $(D$. domestica) that is a member of the same genus as the Virginia opossum (Hunt et al. 2009). In comparing the gene sequences for the LWS opsin gene, they isolated in the context of what had previously been learned about the identity of the amino acid residues associated with spectral tuning in other mammalian species they concluded that $D$. domestica would be predicted to 
have an LWS pigment with peak at about $555 \mathrm{~nm}$. This inferred value is close to that we identified in the Virginia opossum. Whether the modest residual difference (555 vs. $561-562 \mathrm{~nm}$ ) reflects a true species difference or might in some way be associated with differences that reflect some idiosyncrasies of the two estimation techniques is uncertain. In any case, the long-wavelength pigment inferred for the South American opossums is similar, if not identical, to that reflected in the electrophysiological recordings made on this North American species.

Two features of the opossum eye that might be thought to influence these spectral sensitivity measurements deserve brief mention. First is the possibility of some selective spectral filtering by the lens. There are no available measurements of lens transmission in this species but short-wavelength filtering by the lens would be expected to be modest in a strongly nocturnal species such as this one (Kennedy and Milkman 1956) and, in particular, would likely have not much or no effect on the functions of Fig. 1 that extend downward into the short wavelengths only to $460 \mathrm{~nm}$. A second potential factor that might influence spectral sensitivity is the presence in the opossum eye of a retinal tapetum that covers a large fraction of the superior half of the eye giving this species a prominent eye shine. The reflecting material in the opossum tapetum consists of lipid spheres scattered in epithelial cells (Schwab et al. 2002; Ollivier et al. 2004). The reflective properties of this tapetum have not been measured, but to us the tapetal reflection from opossum eyes does not appear obviously colored. In an early examination, Gordon Walls described the opossum tapetum as being "slightly yellowish" in appearance (Walls 1939), but recent fundus photographs give no hint of any coloration (Schwab et al. 2002; Ollivier et al. 2004). If there is any influence of tapetal reflection on the spectral sensitivity of the opossum it is probably minimal.

Two observations made on the retina of the South American opossum D. auritai suggest that the retina of the Virginia opossum might likely be expected to contain a second, short-wavelength sensitive cone pigment. First, the monoclonal antibody OS-2 has been shown to selectively label short-wavelength sensitive cones in the retinas of a number of different mammalian species (Szel et al. 1996) and that marker was used to detect a small population of cones in D. aurita (Ahnelt et al. 1995). Second, the coding sequence for the SWS1 gene recently determined for this same species of opossum has been expressed and regenerated in vitro and absorbance measurements revealed that it was linked to a pigment with $\lambda_{\max }$ of $\sim 362 \mathrm{~nm}$ (Hunt et al. 2009). If one assumes that such a UV pigment may also be present in the Virginia opossum, our failure to detect its presence in ERG recordings could have a number of explanations. Perhaps the most likely one has to do with the sparsity of cones and their consequent feeble contribution to the ERG. Opsin immunolabeling indicates that the overall density of cones in $D$. aurita is not more than $\sim 2,500$ per $\mathrm{mm}^{2}$ while those believed to be short-wavelength sensitive do not exceed $\sim 200$ per $\mathrm{mm}^{2}$ (Ahnelt et al. 1995). By way of comparison, corresponding values for cone representation in the retina of a nocturnal rodent, the pigmented rat, an animal from which signals from both $\mathrm{M}$ and UV cones are easily detectable in the ERG are, respectively, $\sim 7,000$ per $\mathrm{mm}^{2}$ and $700-800$ per $\mathrm{mm}^{2}$ (Jacobs et al. 2001; Ortin-Martiniz et al. 2010).

What functional roles might cones play in the vision of the opossum? An early behavioral experiment claimed to demonstrate the presence of color vision in the Virginia opossum (Friedman 1967). However, the lights among which color discriminations were tested in that experiment all lay in the visible part of the spectrum, a range over which we could detect the presence of only a single cone pigment and such an arrangement would obviate color vision. The rather coarse variation in stimulus intensity used in the early behavioral experiment suggests the alternative interpretation that the successful discriminations among the various colored lights may well have been based on the use of brightness cues. On examining the opossum retina, Walls suggested that, "the sparse cone populations cannot mean much to the animals" (Walls 1939). Whether these cones merely represent a vestigial hangover of the sauropsid past of this species, as Walls was led to conclude, or whether the cones continue to serve an adaptive function that is sufficient to maintain their presence could only be established by direct and careful behavioral examinations. At the least, our observations on the ERG in this species suggest that cone-based vision in this animal is apt to be considerably limited and, more broadly, that it is probably unwarranted to assume that the presence of two types of opsin genes and/or two classes of labeled cones in the nocturnal opossums necessarily implies the presence of a useful dichromatic color vision capacity.

Acknowledgments We thank Jess Deegan II and Jack Calderone for their participation in some of the recording experiments.

Open Access This article is distributed under the terms of the Creative Commons Attribution Noncommercial License which permits any noncommercial use, distribution, and reproduction in any medium, provided the original author(s) and source are credited.

\section{References}

Ahnelt PK, Hokoc JN, Rohlich P (1995) Photoreceptors in a primitive mammal, the South American opossum, Didelphis marsupalis aurita: characterization with anti-opsin immunolabeling. Vis Neurosci 12:793-804 
Arrese CA, Hart NS, Thomas N, Beazley LD, Shand J (2002) Trichromacy in Australian marsupials. Curr Biol 12:657-660

Arrese CA, Oddy AY, Runham PB, Hart NS, Shand J, Hunt DM, Beazley LD (2005) Cone topography and spectral sensitivity in two potentially trichromatic marsupials, the quokka (Setonix brachyurus) and quenda (Isodon obesulus). Proc R Soc Lond B 272:791-796

Arrese CA, Beazley LD, Neumeyer C (2006) Behavioural evidence of marsupial trichromacy. Curr Biol 16:R193-R194

Cowing JA, Arrese CA, Davies WL, Beazley LD, Hunt DM (2008) Cone visual pigments in two marsupial species: the fat-tailed dunnart (Sminthopsis crassicaudata) and the honey possum (Tarsipes rostratus). Proc R Soc Lond B 275:1491-1499

Crescitelli F (1958) The natural history of visual pigments. Ann NY Acad Sci 74:230-255

Davies WL, Carvalho LS, Cowing JA, Beazley LD, Hunt DM, Arrese C (2007) Visual pigments of the platypus: a novel route to mammalian colour vision. Curr Biol 17:R161-R163

Friedman H (1967) Colour vision in the Virginia opossum. Nature 213:835-836

Govardovskii VI, Fyhrquist N, Reuter T, Kuzmin DG, Donner K (2000) In search of the visual pigment template. Vis Neurosci 17:509-528

Hemmi JM (1999) Dichromatic colour vision in an Australian marsupial, the tammar wallaby. J Comp Physiol A 185:509515

Hemmi JM, Maddess T, Mark RF (2000) Spectral sensitivity of photoreceptors in an Australian marsupial, the tammar wallaby (Macropus eugenii). Vis Res 40:591-599

Hisatomi O, Tokunaga F (2002) Molecular evolution of proteins involved in vertebrate phototransduction. Comp Biochem Physiol B 133:509-522

Hunt DM, Chan J, Carvalho LS, Hokoc JN, Ferguson MC, Arrese CA, Beazley LD (2009) Cone visual pigments in two species of South American marsupials. Gene 433:50-55

Jacobs GH (1993) The distribution and nature of colour vision among the mammals. Biol Rev 68:413-471

Jacobs GH (2008) Primate color vision: a comparative perspective. Vis Neurosci 25:619-633

Jacobs GH (2009) Evolution of colour vision in mammals. Phil Trans R Soc Lond B 364:2557-2567

Jacobs GH, Deegan JF II (1992) Cone photopigments in nocturnal and diurnal procyonids. J Comp Physiol A 171:701-707

Jacobs GH, Neitz J, Krogh K (1996) Electroretinogram flicker photometry and its applications. J Opt Soc Am A 13:641-648

Jacobs GH, Fenwick JA, Williams GA (2001) Cone-based vision of rats for ultraviolet and visible lights. J Exp Biol 204:2439-2446
Keller LSF, Drozdowicz CK, Rice L, Bowman TA, Lang CM (1988) An evaluation of three anaesthetic regimes in the gray shorttailed opossum (Monodelphis domestica). Lab Anim 22:269-275

Kennedy D, Milkman RD (1956) Selective light absorption by the lenses of lower vertebrates and its influence on spectral sensitivity. Biol Bull 111:375-386

Kolb H, Wang H (1985) The distribution of photoreceptors, dopaminergic amacrine cells and ganglion cells in the retina of the North American opossum (Didelphis virginiana). Vis Res 25:1207-1221

Macdonald D (ed) (2001) The new encyclopedia of mammals. Oxford University Press, Oxford

Ollivier FJ, Samuelson DA, Brooks DE, Lewis PA, Kalberg ME, Komaromy AM (2004) Comparative morphology of the tapetum lucidum (among selected species). Vet Ophthalmol 7:11-22

Rushton WAH (1972) Pigments and signals in colour vision. J Physiol 220:1P-31P

Schwab IR, Yuen CK, Buyuckmithei NC, Blankenship RN, Fitzgerald PG (2002) Evolution of the tapetum. Trans Am Ophthalmol Soc 100:187-200

Strachan J, Chang L-YE, Wakefield MJ, Marshall Graves JA, Deeb SS (2004) Cone visual pigments of the Australian marsupials, the stripe-faced and fat-tailed dunnarts: sequence and inferred spectral properties. Vis Neurosci 21:223-229

Szel A, Rohlich P, Caffe AR, van Veen T (1996) Distribution of cone photoreceptors in the mammalian retina. Microsc Res Tech $35: 445-462$

Ortin-Martiniz A, Jimenez-Lopez M, Nadal-Nicholas FM, SalinasNavarro M, Alarcon-Martinez L, Sauve Y, Villegas-Perez MP, Vidal-Sanz M, Agudo-Barrioso M (2010) Automatic quantification and topographical distribution of the whole population of $\mathrm{S}$ and $\mathrm{L}$ cones in the adult albino and pigmented rats. Invest Ophthalmol Vis Sci (in press)

Wakefield MJ, Anderson M, Chang E, Wei K-J, Kaul R, Marshall Graves JA, Grutzner F, Deeb SS (2008) Cone visual pigments of monotremes: filling the phylogenetic gap. Vis Neurosci 23:257264

Walls GL (1939) Notes on the retinae of two opossum genera. J Morphol 64:67-87

Wikler KC, Rakic P (1990) Distribution of photoreceptor subtypes in the retina of diurnal and nocturnal primates. J Neurosci 10:3390 3401

Yokoyama S (2000) Molecular evolution of vertebrate visual pigments. Prog Ret Eye Res 19:385-419

Yokoyama S (2008) Evolution of dim-light and color vision pigments. Ann Rev Genom Hum Genet 9:259-282 